\section{Drs. Cron and Chatham reply}

To the Editor:

We appreciate the interest expressed by Dr. C.A. Moura and colleagues in our editorial regarding the role of the rheumatologist during the coronavirus disease 2019 (COVID-19) pandemic ${ }^{1}$. The letter by C.A. Moura, et al emphasizes the need to understand mechanisms of disease underlying the more serious complications of COVID-19 infection, as well as to provide the best treatment possible to large numbers of seriously ill patients during a global pandemic ${ }^{2}$. Many of the treatments administered to date under crisis conditions in uncontrolled fashion have been based upon at least some mechanistic rationale as reflected in the Table 1 accompanying their letter. Well-designed randomized controlled trials (RCT) take time to design, undergo ethics board review, and enroll, and some of these are beginning to result. While the US National Institutes of Health-sponsored RCT with remdesivir has been reported to shorten the time to recovery and duration of hospitalization ${ }^{3}$, it is becoming increasingly apparent that the addition of immunomodulation will likely be required to forestall progression of respiratory failure, treat underlying vascular inflammation, and significantly affect survival ${ }^{1,4}$. Until definitive RCT can be completed, non-randomized cohort studies may help to inform which and at what stage of disease currently available immune-modulating therapies are likely to be helpful. To date, such studies indicate that antimalarials such as hydroxychloroquine do not appear to significantly affect either the need for invasive mechanical ventilation or survival'; whether their known effect on Toll-like receptor signaling might be of benefit in earlier prehospital stages of disease remains to be determined in the context of current RCT. As noted by C.A. Moura, et al , recently published small cohort control studies report encouraging results with Janus kinase (JAK) inhibitors ${ }^{6}$ and anti-interleukin 6 receptor (IL-6R) monoclonal antibodies ${ }^{7}$ regarding decreasing the need for mechanical ventilation and survival. Published cohort studies showing similar encouraging results with anakinra (IL-1R antagonist) are also emerging and would be an appropriate addition to the table ${ }^{8}$. Collectively, these published experiences can help guide both the care of seriously ill patients in settings where trial enrollment is not an option, as well as the design of RCT that will provide the best "calculation of the probability" of the utility of immune modulators in managing severe complications of COVID-19.

As suggested by C.A. Moura, et al, there is likely an appropriate window of opportunity to obtain maximal benefit of immunomodulatory therapy ${ }^{2}$. While up to $25 \%$ of SARS-CoV-2-infected adults are asymptomatic, the early clinical features of COVID-19 infection include constitutional symptoms, fever, headache, and malaise, and as SARS-CoV-2 sets up "shop" in
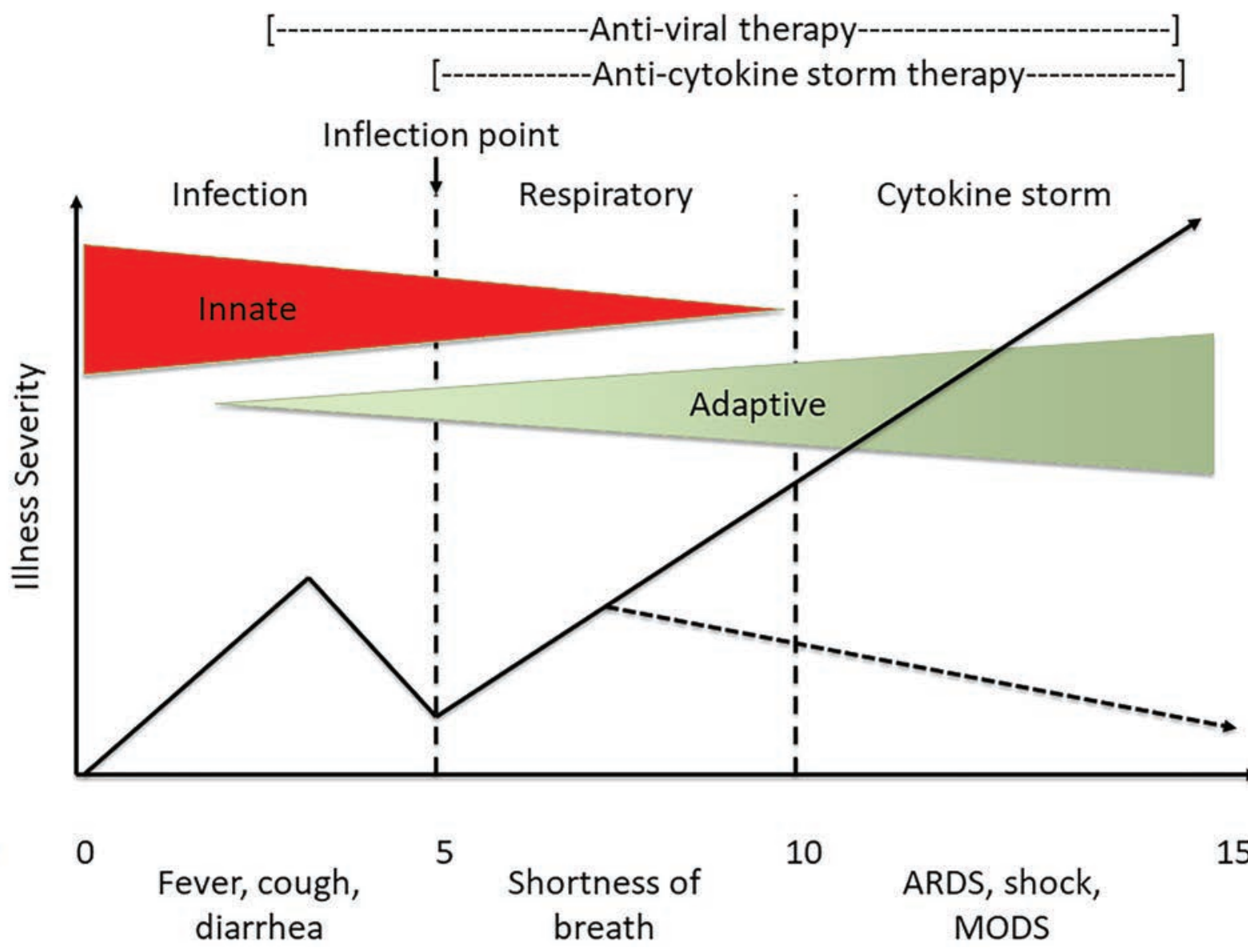

Figure 1. The COVID-19 inflection point of illness. A graphic depiction of the course of illness, for the up-to-20\% of COVID-19 individuals who develop a cytokine storm syndrome (CSS) and respiratory distress requiring hospitalization. Severity of illness is presented along the Y-axis and time in days along the $\mathrm{X}$-axis. The participation of both the innate and adaptive immune responses is presented as respective triangles during different phases of disease. The early stages of infection ( $<5$ days of symptoms) give rise to a more prominent respiratory phase in those with early signs of CSS, such that an inflection point of illness occurs typically between days 5 and 7 of illness. This is the time in which targeted immunomodulatory therapy will likely be most beneficial to lower mortality (dashed arrow). ARDS: acute respiratory distress syndrome; MODS: multiorgan dysfunction syndrome; COVID-19: coronavirus disease 2019. 
the lower respiratory tract, cough can be an early manifestation of disease. For many of these symptomatic COVID-19 individuals, the illness may temporarily wane between days 3 and 5 following symptoms. Somewhere between days 5 and 7 of illness (Figure 1), there is an inflection point where the disease worsens, with some developing a more significant flu-like illness that can be ridden out at home, and others becoming acutely hypoxic, necessitating hospitalization". While the innate immune response is triggered early on, the adaptive immune system has already been engaged by the time that this more severe respiratory stage of disease emerges (Figure 1). In those who are developing acute respiratory distress syndrome, there is likely a cytokine storm syndrome (CSS) evolving from an ongoing interaction of the innate and adaptive immune cells resulting in an inappropriate amplification of proinflammatory cytokines ${ }^{10}$. It is at this stage, just prior to the need for intubation/mechanical ventilation and intensive care, that immunomodulatory approaches should be strongly considered, particularly in those hypoxic COVID-19 patients with laboratory features of CSS, such as hyperferritinemia and elevated C-reactive protein, $\mathrm{D}$-dimer, and lactate dehydrogenase ${ }^{1,4}$.

In addition to potentially effective antiviral treatments, the addition of CSS-directed therapies at the inflection point of disease has the greatest potential to decrease mortality from COVID-19. Which of the various proposed anti-CSS therapies will be most beneficial remains unclear at present prior to RCT results. However, promising reports of small- and moderate-sized case series and cohort comparison studies suggest that several targeted approaches to diminish proinflammatory cytokines will be effective at reducing COVID-19 mortality, particularly when given at the inflection point between the antiviral immune response and the beginning of the CSS. Rheumatologists can help their clinical colleagues determine which hospitalized COVID-19-infected patients are developing a CSS, and how best to manage the CSS with immunomodulation to help save lives ${ }^{1}$.

W. Winn Chatham ${ }^{1}, \mathrm{MD}$, Professor of Medicine

Randy Q. Cron ${ }^{2}$ (I) MD, PhD, Professor of Pediatrics

${ }^{1}$ Department of Medicine, Division of Clinical Immunology and

Rheumatology;

${ }^{2}$ Department of Pediatrics, Division of Rheumatology, University of

Alabama at Birmingham, Birmingham, Alabama, USA.

Drs. Cron and Chatham are co-principal investigators on an investigator-initiated clinical trial to study interleukin 1 blockade in treating secondary HLH in children and adults. The trial is funded by Swedish Orphan Biovitrum Inc. (SOBI; ClinicalTrials.gov: NCT02780583), which manufactures anakinra. Dr. Cron serves as a consultant to SOBI.

Dr. Chatham has served as a consultant to SOBI.

Address correspondence to Dr. R. Cron, Children's of Alabama, Division of Rheumatology, 16007 th Ave. S., CPPN, Suite G10, Birmingham, AL 35233-1711, USA. Email: rcron@peds.uab.edu.

\section{REFERENCES}

1. Cron RQ, Chatham WW. The rheumatologist's role in COVID-19. J Rheumatol 2020;47:639-42.

2. Moura CA, Moura CG, de Sant'Ana Costa AL. SARS-CoV-2: viral mechanisms and possible therapeutic targets - what to learn from rheumatologists. J Rheumatol 2020;47:1588-9.

3. Hendaus MA. Remdesivir in the treatment of coronavirus disease 2019 (COVID-19): a simplified summary. J Biomol Struct Dyn 2020 May 20 (E-pub ahead of print).

4. Henderson LA, Canna SW, Schulert GS, Volpi S, Lee PY, Kernan $\mathrm{KF}$, et al. On the alert for cytokine storm: immunopathology in COVID-19. Arthritis Rheumatol 2020 Apr 15 (E-pub ahead of print).

5. Mahevas M, Tran VT, Roumier M, Chabrol A, Paule R, Guillaud $\mathrm{C}$, et al. Clinical efficacy of hydroxychloroquine in patients with COVID-19 pneumonia who require oxygen: observational comparative study using routine care data. BMJ 2020 May 14 (E-pub ahead of print).

6. Cantini F, Niccoli L, Matarrese D, Nicastri E, Stobbione P, Goletti D. Baricitinib therapy in COVID-19: a pilot study on safety and clinical impact. J Infect 2020 Apr 23 (E-pub ahead of print).

7. Xu X, Han M, Li T, Sun W, Wang D, Fu B, et al. Effective treatment of severe COVID-19 patients with tocilizumab. Proc Natl Acad Sci U S A 2020;117:10970-5.

8. Cavalli G, De Luca G, Campochiaro C, Della-Torre E, Ripa M, Canetti D, et al. Interleukin-1 blockade with high-dose anakinra in patients with COVID-19, acute respiratory distress syndrome, and hyperinflammation: a retrospective cohort study. Lancet Rheumatol 2020 May 7 (in press).

9. Siddiqi HK, Mehra MR. COVID-19 illness in native and immunosuppressed states: a clinical-therapeutic staging proposal. J Heart Lung Transplant 2020;39:405-7.

10. Crayne CB, Albeituni S, Nichols KE, Cron RQ. The immunology of macrophage activation syndrome. Front Immunol 2019;10:119. 